\title{
Potential role of dietary lipids in the prophylaxis of some clinical conditions
}

\author{
Urvashi Bhagat, Undurti N. Das
}

Asha Nutrition Sciences, Inc., Palo Alto, USA

Submitted: 15 March 2014

Accepted: 20 April 2014

Arch Med Sci 2015; 11, 4: 807-818

DOI: 10.5114 /aoms.2015.53302

Copyright (c) 2015 Termedia \& Banach

\section{Abstract}

An imbalance of dietary lipids may potentially have a significant role in the pathobiology of some chronic diseases. Public health dietary fat recommendations have emphasized that low saturated fat, high monounsaturated fat, and high polyunsaturated fat with a lower $\omega-6$ to $\omega-3$ fatty acid ratio intake are necessary for normal health. However, such universal recommendations are likely to be hazardous, since the outcome of recommended lipid intake may depend on the consumption of other important dietary constituents that have an important role in the metabolism of lipids. In addition, consumption of fatty acids as per the individually tailored specific requirements in the context of other nutritional factors may have the potential to stabilize hormones, mood and sleep, and minimize adverse events. In support of this proposal, we review various factors that influence fatty acid metabolism, which need to be taken into consideration for appropriate utilization and consequently prevention of various diseases.

Key words: prevention, fatty acids, antioxidants, phytochemicals, inflammation, cytokines, unsaturated fatty acids, prostaglandins.

\section{Introduction}

Both qualitative and quantitative imbalances in the intake and metabolism of dietary fats have been implicated in a number of chronic diseases including cardiovascular diseases (CVD), obesity, diabetes mellitus (DM), and rheumatoid arthritis (RA) [1-5]. Thus far, in order to overcome these imbalances, the suggested preventative solutions have focused on the delivery of one or more lipids in the form of supplementation of $\omega-3$ fatty acids [2], conjugated-linoleic acid (LA) [6], and $\gamma$-linolenic acid [7]; and other recommendations include enhanced use of certain oils, such as olive oil and canola oil, in order to deliver greater amounts of monounsaturated fatty acids and $\alpha$-linolenic acid $[1,2]$. Reduction in saturated fatty acid consumption has also been recommended [1, 3, 4]. Though these broad health recommendations appear to have reduced the risk of some diseases, they are not uniformly beneficial and in fact, may actually enhance the risk of some disease. For instance, it was reported that replacing dietary saturated fat with $\omega-6$ linoleic acid, for the secondary prevention of coronary heart disease and death, showed no evidence of cardiovascular benefit [8]. This may be interpreted to mean that other dietary components that are essential for its (LA) beneficial action also need to be obtained to derive the beneficial action of increased

\author{
Corresponding author: \\ Urvashi Bhagat \\ Undurti N. Das, MD, FAMS, \\ FRSC \\ Asha Nutrition Sciences Inc. \\ P.O. Box 1000, Palo Alto \\ CA 94302, USA \\ Phone/fax: 650-322-7861 \\ E-mail: \\ bhagatu@asha-nutrition.com, \\ Undurti@hotmail.com
}


consumption of LA. It is noteworthy that LA is oxidized to form oxidized LA metabolites (OXLAMs) that are the most abundant oxidized fatty acids in oxidized low density lipoprotein, which are potentially more atherogenic than unmodified low density lipoprotein. This implies that various factors that have a modulatory influence on LA metabolism such as antioxidants [9-12], phytochemicals [13-18], minerals [19], gender [20, 21], age [22], and genetics [23] play a significant role in bringing about its (LA) beneficial action. Thus, there are many variables that modulate the metabolism of various fatty acids. Furthermore, it will be difficult for consumers to calibrate on a daily basis the demands of the body for various fatty acids. This is so since the requirements of various biologically active unsaturated fatty acids change depending on age, gender, and various life style factors. It is possible that there could exist differences in the requirements of various fatty acids and their co-factors even among members of the same family. In view of this, it is important to evolve precise personalized yet broad based dietary lipid program(s) that are easy to implement to prevent various diseases. It is the purpose of this review to discuss various factors that influence fatty acid metabolism based on which guidelines to develop customized lipid programs can be drawn and recommended.

\section{Metabolism of essential fatty acids}

Dietary lipids include fatty acids, sterols, carotenoids, and vitamins $A$ and $E$. A good review of the terminology, sources, digestion, metabolism, and physiological actions of lipids is provided by Ratnayake and Galli [24]. In summary, linoleic acid (LA, C18:2) and $\alpha$-linolenic acid (ALA, C18:3) are essential fatty acids (EFA) since humans cannot synthesize them de novo but they are essential for survival. Though both LA and ALA are biologically active $[1,24]$, they need to be converted to their long-chain metabolites to gain benefit of their full potential $[25,26]$. Linoleic acid, the $\omega-6$ EFA, is elongated and desaturated to give rise to its longchain metabolites: $\gamma$-linolenic acid (GLA, C18:3), dihomo-gamma-linolenic acid (DGLA, C20:3), and arachidonic acid (AA, C20:4). Dihomo-gamma-linolenic acid forms the precursor of 1 series prostaglandins (PGs), whereas AA is the precursor of 2 series PGs, thromboxanes (TXs), and 4 series leukotrienes (LTS). On the other hand, ALA is the precursor of its long-chain metabolites eicosapentaenoic acid (EPA, C20:5) and docosahexaenoic acid (DHA, C22:6) of the n-3 family. Eicosapentaenoic acid gives rise to 3 series PGs and TXs, and 5 series LTs. LA, GLA, DGLA, AA, ALA, EPA, and DHA are all polyunsaturated fatty acids (PUFA), while only LA and ALA are EFAs. All EFAs are also PUFAs but all PUFAs are not EFAs. Eicosanoids (PGs, TXs and LTs) have many actions and are involved in several physiological and pathological processes, some of which include: blood vessel constriction, dilation, blood pressure regulation, platelet aggregation, and modulation of inflammation, etc. [24, 27]. In general, eicosanoids derived from AA have more potent actions compared to those derived from EPA, though there are exceptions to this generalization [28]. Additionally, AA, EPA, and DHA are precursors to lipoxins, resolvins, and neuroprotectins that have potent anti-inflammatory actions [24]. Polyunsaturated fatty acids and their products including eicosanoids, lipoxins, resolvins and protectins modulate a number of biological functions by their ability to form an active component of cell membranes and by influencing pinocytosis, ion channel regulation and gene expression [24, 27].

$\alpha$-Linolenic acid, LA, and oleic acid (OA) undergo oxidative desaturation by the same set of enzymes - delta-6-desaturase $\left(\Delta^{6}\right)$ and delta-5-desaturase $\left(\Delta^{5}\right)$ - to give rise to their respective - PUFAs $[29,30]$. Among the three fatty acids, ALA is preferentially desaturated, LA second, and OA third (ALA > LA > OA) $[29,31]$. This affinity of the $\Delta^{6}$ and $\Delta^{5}$ to their substrate has important therapeutic implications since changes in the availability and/or increases or decreases in one substrate can have a profound effect on the metabolism of the other substrates. In view of this, consumption of the right balance of dietary fatty acids is important and this constituted a vigorous debate as to what is the right proportion or ratio of $n-3, n-6$ and $n-9$ to be consumed for their optimal utilization and usefulness in the body. A number of studies have discussed the importance of maintaining a balance between $\omega-6$ and $\omega-3$ fatty acids in human nutrition for optimal function of various tissues specifically taking into consideration the eicosanoids produced from $\omega-6$ and $\omega-3$ fatty acids due to their significant divergent actions especially in inflammation, among other reasons [32-34]. The present pattern of consumption indicates that the ratio between $\omega$-6-to- $\omega-3$ ratios is $\sim 15-17: 1$ in Western diets, which has been cited as one of the important dietary factors that has led to the increase in the incidence of modern chronic diseases such as insulin resistance, atherosclerosis, type 2 diabetes mellitus and cancer [2, 33].

For example, studies have shown that adult human brain consumes $\mathrm{AA}$ and DHA at rates of 17.8 and $4.6 \mathrm{mg} /$ day, respectively (ratio $-3.87: 1$ ), respectively [35]. Further, it was shown that most adult human tissue contains approximately 10 times AA as compared to DHA [36]. This demonstrates that $A A$ requirement is 4 to 10 times that of DHA. Furthermore, it has been shown to be 
equally competitive, LA and ALA should be in the ratio of $14: 1$ [32]. Based on this logic the ratio between $\omega-6$-to- $\omega-3$ of $15-17: 1$ in diets is not the problem, the problem is the other factors that influence the metabolism of $\omega-6$ and $\omega-3$.

A substantial number of studies revealed additional complexity in the metabolism of essential fatty acids and their long-chain metabolites besides the complexity that exists as a result of changes in the ratios among fatty acids. For instance, previously we noted that when Sprague-Dawley rats (200-220 g) were fed a fatfree semi-synthetic diet supplemented with $10 \%$ (by weight) of different combinations of evening primrose oil (EPO), a rich source of LA and $\gamma$-linolenic acid, and polepa (POL), a marine oil rich in eicosapentaenoic (EPA) and docosahexaenoic (DHA) acids (the combinations of supplement were $9 \%$ EPO-1\% POL, 8\% EPO-2\% POL, 7\% EPO-3\% POL, $6 \%$ EPO-4\% POL and 5\% EPO-5\% POL) it was observed that animals fed higher proportions of POL consistently contained higher levels of DGLA $(p<0.05)$ and lower levels of AA $(p<0.05)$. Thus, an inverse relationship between AA/DGLA ratio and EPA levels was found to exist $(r=-0.765$ in plasma and -0.792 in liver [37]. In a similar fashion, an interaction may occur between ALA/EPA ratio and AA levels. Such an interaction among various $n-3$ and $n-6$ fatty acids makes it difficult to anticipate how the metabolism of PUFAs and formation of various eicosanoids occur and at times difficult to foresee and predict the products that are likely to be formed from various PUFAs under different physiological and pathological conditions. Despite this complex interaction among various $n-3$ and $n-6$ fatty acids, certain generalizations are possible, though arriving at some of these conclusions needs to be done rather cautiously. This implies that in all clinical conditions multiple changes in the concentrations of plasma and/or tissue fatty acid profile may occur and no single fatty acid could serve as a marker of any particular disease. For instance, it was reported that high proportions of palmitic acid (16:0), palmitoleic acid (16:1), and DGLA, and a low proportion of LA, AA, EPA and DHA, occur in the plasma/serum in type-2 diabetes [3, 34, 38, 39], myocardial infarction [40, 41], stroke [42], left ventricular hypertrophy [43], and metabolic syndrome [44, 45]. Increased activity of SCD (stearoyl CoA desaturase, also known as delta-9-desaturase, which desaturates saturated fatty acids (SFA) to form monounsaturated fatty acids (MUFA)), and low $\Delta^{5}$ activity have also been described to be independently associated with increased risk for cardiovascular diseases, insulin resistance and low-grade systemic inflammation, and cardiovascular and total mortality [46, 47]. Warensjo et al. observed an independent asso- ciation between desaturase activity indices and mortality risk. They suggested that altered endogenous desaturation might contribute to the risks [47]. Others have suggested that a defect in $\Delta^{6}$ and $\Delta^{5}$ may be a factor in the initiation and progression of insulin resistance and atherosclerosis and their associated diseases such as obesity, diabetes mellitus, and hypertension [3, 40, 48, 49]. The complexity of the involvement of PUFAs in various diseases is further evident from the studies with regard to the role of linoleic acid (LA, 18:2n-6) and its metabolites specifically in cardiovascular diseases. For instance, studies performed both in experimental animals and humans in the early 1950s and 1960s showed that increased intake of saturated fats increases plasma cholesterol levels and leads to the development of hypercholesterolemia, while enhanced intake of unsaturated fatty acids including LA reduces plasma and tissue cholesterol levels [50-56].

\section{Factors influencing the metabolism of essential fatty acids}

It is well documented that various dietary and non-dietary factors modulate the metabolism of EFAs and consequently the formation of various eicosanoids. Some of these factors include: the ratio among various polyunsaturated fatty acids, antioxidants, phytochemicals, vitamins, minerals, hormones (especially estrogen, insulin, corticosteroids), gut microbiota, ethanol, oncogenic viruses, and genetics and age of the individual, and climactic temperature [57-62]. A brief description as to the way some of these factors influence EFA metabolism is given below (see also Figure 1).

\section{Modulators of desaturases}

It is well recognized that $\Delta^{6}$ and $\Delta^{5}$ desaturases are the rate-limiting factors in the production of long-chain metabolites of EFAs: LA and ALA [6365]. Hence, factors that influence the activities of desaturases are expected to alter the tissue levels of LA and ALA and their long-chain metabolites. Thus, several nutritional, hormonal, and genetic factors are able to determine the plasma and tissue concentrations of various PUFAs as a result of their influence on the activity of desaturases [30, 66]. $\Delta^{6}$ desaturase activity is upregulated by EFA, protein, insulin and dietary deficiency (calorie restriction), and downregulated by fasting, glucose, fructose, glycerol, EFA excess, metabolic hormones (other than insulin), ethanol and increasing age $[29,30,57-66] . \Delta^{5}$ desaturase responds similarly to metabolic hormones, but in the event of EFA deficiency it is downregulated rapidly and is upregulated with increase in the activity of $\Delta^{6}$ desaturase, showing a delayed response. On the other hand, 


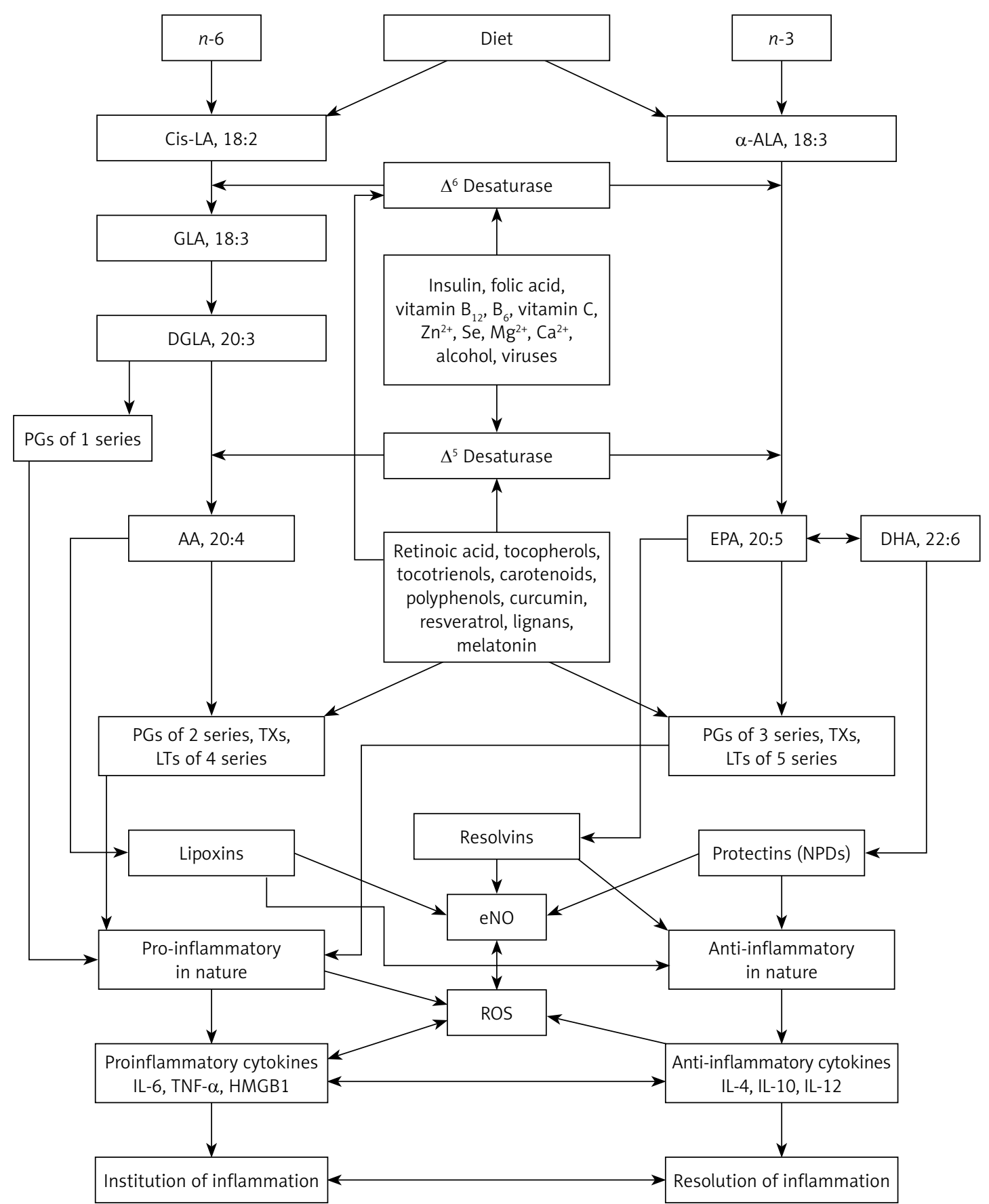

Figure 1. Scheme showing the metabolism of essential fatty acids, their role in inflammation and factors that influence desaturases

in response to an increase in EFA consumption (or increased release of EFAs from the cell membrane pool) the activity of $\Delta^{6}$ desaturase declines whereas that of $\Delta^{5}$ desaturase is increased $[66,67]$. Once the feedback regulation comes into play, the activities of both the desaturases are restored to normal [30]. This may explain why a rapid increase in the consumption of $\omega-6$ fatty acids by subjects who are deficient in these fatty acids ( $n-6$ PUFAs) results in a sudden surge in the features of inflammation that could be attributed to increased formation of pro-inflammatory eicosanoids derived from $n-6$.
Males and females seem to differ in their ability to synthesize long-chain $\omega$-3 fatty acids from ALA as a result of the action of estrogen and testosterone on its (ALA) metabolism. Estradiol increases, whereas testosterone decreases the production of long-chain metabolites derived from LA and ALA $[20,21]$. It has been reported that the $\omega-3$ pathway is more responsive to hormonal treatment than the $\omega-6$ pathway; relatively low concentrations of estradiol increased the synthesis of EPA and docosapentaenoic acid (DPA) from ALA, but larger concentrations of estradiol were required to 
increase the AA levels [68]. A lower partitioning of ALA for $\beta$-oxidation and a lower use as an energy source in women compared with men has also been reported, which may enhance its availability for the formation of EPA and DHA [69]. This could be one specific reason why greater DHA synthesis has been reported in women than men, which may result in higher plasma DHA concentration in women [70]. In females, the conversion from ALA to DHA may be as high as $9 \%$, whereas for males it may be $\sim 0.5-4 \%$ [70]. Growth hormones have been shown to increase the $\Delta^{6}$ activity and consequently enhance the tissue levels of respective PUFAs in the tissues [71].

Vitamin A has been shown to downregulate the expression of $\Delta^{5}$ [72]. In addition, some phytochemicals, particularly curcumin and sesamin, also downregulate $\Delta^{5}$. But, surprisingly, both curcumin and sesamin suppressed desaturation of $\omega-6$ fatty acids but not of $\omega-3$ fatty acids $[16,73]$. Curcumin is more effective than sesamin, while simultaneous use of both curcumin and sesamin had a greater suppressive effect on chain elongation, resulting in tissue accumulation of GLA and DGLA. In contrast, phytosterols enhanced the activity of $\Delta^{6}, \Delta^{5}$, and SCD [74]. Stearoyl CoA desaturase by virtue of its ability to introduce a double bond in SFA to form MUFA increases the unsaturation index and thus cell membrane fluidity (Figure 1).

The effect of temperature on the activity of desaturases has been controversial, some reporting an increase in $\Delta^{6}$ activity at lower temperatures, while others report decreased activity [75-78]. It stands to reason to suggest that at low temperatures, the activity of desaturases could be higher, since to maintain cell membrane fluidity in a cold climate higher concentrations of unsaturated fatty acids are needed.

Arguably, one of the important factors that regulate the activity of desaturases is the cellular content of unsaturated fatty acids themselves. Under normal physiological conditions, cellular PUFA content is maintained within a narrow range by the activity of desaturases and elongases and their uptake and efflux. As expected, as is the case with the activity of many other enzymes in the cells, upregulation of the PUFA synthetic pathway occurs principally under conditions of deficiency while, as expected, downregulation of the desaturases and elongases occurs, rather quickly, once PUFAs have been replenished or provided $[27,67]$.

\section{Other fatty acids}

Although non-essential fatty acids can be synthesized endogenously, some of them are considered conditionally essential and they influence EFA metabolism. For example, OA not only has regulatory functions but can also alter cellular fat- ty acid composition in select organs [25]. Both saturated and unsaturated fatty acids are essential components of the cell membrane and contribute to many cellular functions as well. Some of these include: coordinating the expression of proteins involved in lipid synthesis, transport, storage, degradation, and elimination to maintain a normal physiological state $[79,80]$. Several of these fatty acids function as ligands of nuclear and cell-surface receptors and thus maintain cellular homeostasis [26], by sensing cellular lipid levels and regulating gene expression to control lipid overload. The homeostatic role of lipids includes regulation of energy and glucose homeostasis through a feedback regulation between the gastrointestinal tract and central nervous system in which fatty acids with 12 or more carbons seem to have an important role by regulating food intake [81]. This sensitive neuronal circuitry becomes inefficient in response to high-fat or inappropriate fat intake, which could be attributed to imbalance in the ingestion of specific fatty acids [82]. Palmitic, lauric, and stearic acids stimulate the expression of mitochondrial uncoupling proteins, UCP2 and UCP3, which reduce oxidative stress and are known to play a role in determining longevity of the organism [83].

Dietary composition of fatty acids (including $\omega-6$ and $\omega-3)$ is reflected in tissue composition $[84,85]$, which may have a modulatory influence on cellular functions. Similarly, the total amount of dietary fatty acids (low-fat versus high-fat diets) influences fatty acid metabolism and tissue composition. For instance, consumption of low fat diets seems to enhance plasma $\omega-3$ fatty acid levels, which could be due to the preferential metabolism of ALA [86]. Increased intake of a high fat diet, especially saturated fats, can be a risk factor for the development of hypertension [4]. Whether this increase in blood pressure due to high intake of saturated fats may be related to interference with the metabolism of essential fatty acids and/or an imbalance in the formation of their eicosanoid metabolites remains to be established. Nevertheless, it is likely that the proportion of $\omega-6$ and $\omega-3$ fatty acids, saturated fats and concomitant consumption of total protein and carbohydrate may all play a significant role in the pathobiology of hypertension and other cardiovascular diseases [87-92].

\section{Antioxidants, phytochemicals, vitamins and minerals}

Following the ingestion of fatty acids, they may undergo: (1) mitochondrial and peroxisomal $\beta$-oxidation for energy production, (2) free-radical mediated oxidation (chain reactions where one free radical can oxidize many lipid molecules), (3) free-radical independent, non-enzymatic oxi- 
dation, and/or (4) enzymatic oxidation to produce bioactive lipid products including long-chain fatty acids and various eicosanoids. Specific products are formed from each type of oxidation and specific antioxidants are known to modulate specific reaction $[9,93]$. This may explain the regulatory or modulatory role played by several antioxidants, phytochemicals, vitamins, and minerals in the metabolism or bioavailability of various fatty acids.

Vitamin $\mathrm{E}$ and $\mathrm{C}$ work synergistically to prevent lipid peroxidation $[9,94]$. Both cyclooxygenase-2 (COX-2) activity and lipid peroxidation increase with age, which could be inhibited by vitamin $E$ [95-100]. PGE2, a product of COX-2 activity, is an immunosuppressor and so it is anticipated that vitamin $\mathrm{E}$ may be able to restore immune dysfunction and increase T-cell-mediated immune function $[98,99] . \gamma$-Tocopherol $(\gamma T)$ is a more effective inhibitor of $\mathrm{PGE}_{2}, \mathrm{LTB}_{4}$, and tumor necrosis factor- $\alpha$ (TNF- $\alpha$ ) than $\alpha$-tocopherol ( $\alpha$ T) [100]. It is worth noting that with advancing age, production of pro-inflammatory cytokines IL- 6 and TNF- $\alpha$ increases, while both vitamin $\mathrm{E}$ and PUFAs and their eicosanoid products inhibit their (IL- 6 and TNF- $\alpha$ ) synthesis [98-102]. On the other hand, increased production of free radicals and the lipid peroxidation process, which increase with aging, may have an impact on the availability of PUFAs, since the latter are easily peroxidized. Thus, there appears to be close but intricate and complex interaction among vitamin E, PUFAs, eicosanoids, lipid peroxidation and cytokines that ultimately may impact the immune response and various aging associated diseases such as type 2 diabetes mellitus, hypertension, metabolic syndrome, Alzheimer's disease and cancer. Thus, vitamin E requirements are partially dependent on PUFA consumption, partly because PUFAs reduce intestinal absorption of vitamin E [10]. In this context, it is important to note that the results of the GISSI trial [103] reported that vitamin E supplementation does not prevent myocardial infarction and yet other studies showed that both $\beta$-carotenoids and vitamin A intake may actually increase the incidence of cancer in the high-risk population [104]. These results suggest that the timing, dose, and form of administration of anti-oxidants may produce unexpected and contradictory results. Studies have also shown that vitamin A can modulate PUFA metabolism and formation of various eicosanoids $[105,106]$. Folic acid stimulates the formation of long-chain $n-3$ fatty acids [107], which may explain its importance in brain growth and function for which even PUFAs are essential.

Phytochemicals stimulate the synthesis of detoxifying and antioxidant enzymes and may also modulate plasma membrane structure and act as ligands to certain cellular signaling molecules [13,
108-110]. For instance, curcumin accumulates in the plasma membrane and alters thickness, fluidity, and elasticity, whereas resveratrol increases membrane fluidity [13].

Melatonin, the circadian rhythm regulator, has the ability to counteract lipid peroxidation in biological membranes and serve as an antioxidant [111]. Long-term melatonin administration reduced hyperinsulinemia and improved the altered fatty-acid compositions in type 2 diabetic rats via the restoration of $\Delta^{5}$ activity, indicating that melatonin can modulate essential fatty acid metabolism [112].

The influence of minerals and trace elements on AA metabolism and eicosanoid production is complex [19]. Selenium, an important component of the Se-dependent enzyme glutathione peroxidase (Se-GSHpx), functions synergistically with vitamin $\mathrm{E}$ as an antioxidant and thus may prevent lipid peroxidation and alter the production of eicosanoids. Zinc, cadmium, silver, iron, and mercury are inhibitors of Se-GSHpx, which is known to catalyze AA metabolism to form PGs, TXs, and LTs. Free radical generated during the formation of various eicosanoids themselves may have a feedback regulatory function on their (eicosanoids) formation [113-115]. Thus, while considering the metabolism of PUFAs and the formation of various eicosanoids, one needs to take into account the presence, actions and concentrations of various antioxidants, phytochemicals, vitamins, and minerals.

The biological role of lipid peroxides is complex, especially with regard to their role in pathological processes and diseases such as diabetes, atherosclerosis, inflammation, aging, and ischemia-reperfusion injury $[9,96]$. It is believed that low to moderate levels of lipid peroxides are essential for cellular functions by triggering adaptive responses that are necessary to prevent cytotoxic actions of oxidative stress by upregulating protective antioxidant defenses [9, 60, 61, 113-115].

\section{Gut microbiota}

Gut microflora can influence lipogenesis and plasma lipopolysaccharide levels [116]. A high-fat diet may have an unfavorable effect on gut microflora [117], while the gut microbiota influences fat composition of host tissue. For instance, administration of Bifidobacterium breve with linoleic acid increased the tissue composition of conjugated-linoleic acid and $\omega-3$ fatty acids EPA and DHA [118]. The effect of other PUFAs on gut microbiota remains to be determined.

\section{Gender, genetics and aging}

Sex hormones can alter metabolism of dietary fats [20,21], while dietary fats modulate the syn- 
thesis of sex hormones and the associated receptor organization [99, 119]. Higher PUFA administration resulted in lower activity of steroidogenic enzymes and low levels of androgens as compared to MUFA or SFA administration. $\omega$-3 fatty acids, particularly DHA, caused less androgen production than $\omega-6$ fatty acids; and $\omega-6$ fatty acids caused less androgen production than MUFA or SFA $(\omega-3>\omega-6>$ MUFA $=$ SFA) [120]. For this alteration in the androgen levels to occur, fatty acids need to be administered for at least 3 weeks, while feeding fats for 6 weeks led to a decrease in androgen, implying that adapter mechanisms come into play when fats are fed for longer periods [121]. A similar relationship that has been shown by androgen production seems to exist between estrogen and PUFAs [122]. Both estrogen and PUFAs enhance nitric oxide synthesis, suppress the production of pro-inflammatory IL- 6 and TNF- $\alpha$ production and have antioxidant-like and anti-atherosclerotic properties, and showed neuroprotective actions [122]. Men and women differ in storage, mobilization, and oxidation of fatty acids [123, 124], and gene expression relevant to fatty acid metabolism [125-127].

Genetic variations in the activity of delta desaturases and elongases can influence metabolism and therefore the requirement and concentrations of cellular lipids [23]. Similarly, polymorphisms in apolipoprotein $\mathrm{E}$ and peroxisome-proliferator-activated receptor- $\gamma$ (PPAR- $\gamma$ ) genes alter the response to dietary fats [126]. On the other hand, dietary fats can alter the expression of several genes. For instance, PUFAs suppress lipogenic, glycolytic, and cholesterologenic genes, but enhance the expression of genes of the $\beta$-oxidation pathway $[127,128]$. The PUFAs modulate gene expression by interacting with nuclear receptors - hepatic nuclear factor (HNF-4), liver $X$ receptors (LXR), and PPAR $\alpha, \beta, \delta$, and $\gamma$ - and by regulating transcription factor sterol regulatory element-binding proteins (SREBP) 1 and 2 [128]. SREBPs, suppressed by PUFAs, are key regulators of cholesterol, fatty acid, and triglyceride synthesis. Linoleic acid and AA are potent PPAR ligands, producing a rapid increase in expression of genes involved in lipid oxidation.

Phytochemicals bind to the cell surface and nuclear receptors as ligands. Curcumin, capsaicin, ginsenosides, hesperidin, and resveratrol are PPAR- $\gamma$ ligands, attenuate cytokine production and thus suppress inflammation [18]. Phytosterols alter expression of intestinal and hepatic genes [129]. Since nutrients are able to alter a variety of genes, it is tempting to suggest that fine tuning of the ingestion of various nutritional factors could be employed to optimize gene expression and thus prevent several diseases.
It is believed that with advancing age membrane fluidity declines, lipid peroxidation increases and so also does oxidative stress. Aging is one of the factors that impact the activity of desaturases, leading to an alteration in the formation of long-chain metabolites of EFAs: LA and ALA. Thus, this could be a compensatory phenomenon - as oxidative stress increases with age, the activity of desaturases changes and the tissues try to maintain near normal amounts of PUFA though they could form substrates for the peroxidation process. But, this delicate balance between oxidative stress and peroxidation on one hand and the activity of desaturases on the other hand may lead to significant alterations in cell membrane fluidity, formation of various eicosanoids, and consequently changes in the formation of cytokines events that could have a profound influence on the immune response and inflammation. Calorie restriction enhances the activity of desaturases, which could be considered as yet another compensatory phenomenon since with aging food intake decreases. Since calorie restriction also extends life span, it is tempting to suggest that the close interaction(s) among oxidative stress of aging, lipid peroxidation, activity of desaturases, formation of various eicosanoids, calorie intake, production of cytokines and consequent alterations in inflammation and immunity may be relevant to the involvement of these changes in a variety of diseases.

It has been suggested that a decline in brain DHA content with age is associated with increased lipid peroxidation [130] that may lead to impaired cognitive function as a result of neuronal apoptosis of the cerebral cortex and hippocampus [131]. Hence, increased consumption of DHA (in the form of fish oil) could be of benefit in dementia of aging, Alzheimer's disease and depression. This suggestion looks paradoxical since one would expect that increased consumption of DHA may enhance the lipid peroxidation process and enhance oxidative stress. But, in practice enhanced DHA consumption failed to increase oxidative stress in humans $[132,133]$, implying that the lipid peroxidation process does not just depend on the amount of unsaturation and is not a non-specific process but could be a specific enzymatic process that depends on local cellular integrity, function, and the necessity of eicosanoids and other products for various physiological and pathological processes.

\section{Conclusions}

It is evident from the preceding discussion that PUFAs not only form an important constituent of the cell membrane but also play an important role in inflammation and immunity. The effect of PUFAs on 
inflammation and immunity depends on the products formed from them. The exact mechanisms that determine what types of products are derived from various PUFAs - pro-inflammatory or anti-inflammatory - is not clear. Both PUFAs and the products formed from these fatty acids may ultimately determine the initiation, continuation and/or resolution of inflammation and the magnitude and type of immune response [134]. Some, if not all, of the actions of PUFAs and their products on inflammation and immunity could be attributed to their action on NF$\kappa \mathrm{B}$, PPARs and other transcription factors.

Though it is not yet certain, it is likely that cellular stores of PUFAs and phytochemicals and other co-factors that alter fatty acid and eicosanoid metabolism play a significant role in several disease processes. It is possible that a sudden withdrawal of or alteration in the proportion of intake of different types of PUFAs may result in a sudden surge in the production or inhibition of certain eicosanoids that may result in unrestrained or significant alterations in production/suppression of cytokines and gene(s) expression that may result in significant alterations in the physiological or pathological processes including changes in LDL, HDL and cholesterol [135-137]. Such sudden and, sometimes, even gradual and unanticipated changes in the concentrations of various PUFAs, eicosanoids, cytokines, oxidative stress, HDL (may make HDL dysfunctional), LDL, cholesterol, triglycerides and other bioactive molecules may render the host vulnerable to infections, myocardial infarction, stroke, and other diseases and their complications [138-157].

In view of this, it is essential to determine the individual necessity of various monounsaturated, $\omega-6, \omega-3$ and other fatty acids, antioxidants, and phytochemicals and administer them accordingly. Such an individualized approach may be more fruitful in tackling several diseases in which PUFAs are believed to play a significant role. Development of such personalized dietary lipid programs for different types of subjects depending on their age, gender, dietary practices, environmental factors (such as temperature, season, etc.), hormonal status, stress and strain of life and other life style factors (such as exercise, etc.) and genetic background is probably necessary and important to derive the best out of PUFAs, phytochemicals, vitamins and other co-factors for optimum health and to ward off diseases.

In this context, it is noteworthy that some of the beneficial actions of statins could be brought about by PUFAs [158] and implies that a combination of statins and PUFAs may be more beneficial to patients with hyperlipidemias including those who have statin intolerance [159].

Such a dietary program should also take into consideration the necessity of saturated, mono- unsaturated, and polyunsaturated fatty acids, phytochemicals, antioxidants, and minerals, such that the body tissues would have access to all the required raw chemicals/ingredients to form the beneficial bioactive compounds to optimize health.

\section{Acknowledgments}

We are grateful to Dr. Kent L. Erickson, Professor, Department of Cell Biology and Human Anatomy, University of California, School of Medicine, Davis, California, USA, for constructive comments and editing of the manuscript.

\section{Conflict of interest}

Urvashi Bhagat is CEO of Asha Nutrition; Undurti N. Das is the Chief Medical Officer and Chairman of the Scientific Advisory Board of Asha Nutrition. Asha Nutrition has developed and marketed products based on essential fatty acids and other fatty acids.

\section{References}

1. Galli C, Calder PC. Effects of fat and fatty acid intake on inflammatory and immune responses: a critical review. Ann Nutr Metab 2009; 55: 123-39.

2. Simopoulos AP. n-3 fatty acids and human health: defining strategies for public policy. Lipids 2001; 36 Suppl: S83-9.

3. Hodge AM, English DR, O'Dea K, et al. Plasma phospholipid and dietary fatty acids as predictors of type 2 diabetes: interpreting the role of linoleic acid. Am J Clin Nutr 2007; 86: 189-97.

4. Rasmussen BM, Vessby B, Uusitupa M, et al. Effects of dietary saturated, monounsaturated, and n-3 fatty acids on blood pressure in healthy subjects. Am J Clin Nutr 2006; 83: 221-6.

5. Das UN. Molecular basis of health and disease. Springer, New York 2011.

6. DeLany JP, West DB. Changes in body composition with conjugated linoleic acid. J Am Coll Nutr 2000; 19: 487S-93S

7. Kapoor R, Huang YS. Gamma linolenic acid: an antiinflammatory omega-6 fatty acid. Curr Pharm Biotechnol 2006; 7: 531-4.

8. Ramsden CE, Zamora D, Leelarthaepin B, et al. Use of dietary linoleic acid for secondary prevention of coronary heart disease and death: evaluation of recovered data from the Sydney Diet Heart Study and updated meta-analysis. Br Med J 2013; 346: e8707.

9. Niki E. Lipid peroxidation: physiological levels and dual biological effects. Free Radic Biol Med 2009; 47: 469-84.

10. Bassler KH. On the problematic nature of vitamin $E$ requirements: net vitamin E. Z Ernahrungswiss 1991; 30: $174-80$.

11. Meydani SN, Barklund MP, Liu S, et al. Vitamin E supplementation enhances cell-mediated immunity in healthy elderly subjects. Am J Clin Nutr 1990; 52: 557-63.

12. Sujak A, Gabrielska J, Grudzinski W, Borc R, Mazurek P, Gruszecki WI. Lutein and zeaxanthin as protectors of lipid membranes against oxidative damage: the structural aspects. Arch Biochem Biophys 1999; 371: 301-7. 
13. Ding H, Tauzin S, Hoessli DC. Phytochemicals as modulators of neoplastic phenotypes. Pathobiology 2009; 76: 55-63.

14. Terao J, Kawai Y, Murota K. Vegetable flavonoids and cardiovascular disease. Asia Pac J Clin Nutr 2008; 17 Suppl 1: 291-3.

15. O'Leary KA, de Pascual-Tereasa S, Needs PW, Bao YP, O'Brien NM, Williamson G. Effect of flavonoids and vitamin $\mathrm{E}$ on cyclooxygenase-2 (COX-2) transcription. Mutat Res 2004; 551: 245-54.

16. Fujiyama-Fujiwara $Y$, Umeda R, Igarashi O. Effects of sesamin and curcumin on delta 5-desaturation and chain elongation of polyunsaturated fatty acid metabolism in primary cultured rat hepatocytes. I Nutr Sci Vitaminol (Tokyo) 1992; 38: 353-63.

17. Shah BH, Nawaz Z, Pertani SA, et al. Inhibitory effect of curcumin, a food spice from turmeric, on platelet-activating factor- and arachidonic acid-mediated platelet aggregation through inhibition of thromboxane formation and Ca2+ signaling. Biochem Pharmacol 1999; 58: 1167-72.

18. Marion-Letellier R, Dechelotte P, lacucci M, Ghosh S. Dietary modulation of peroxisome proliferator-activated receptor gamma. Gut 2009; 58: 586-93.

19. Peplow PV. Modification to dietary intake of sodium, potassium, calcium, magnesium and trace elements can influence arachidonic acid metabolism and eicosanoid production. Prostaglandins Leukot Essent Fatty Acids 1992; 45: 1-19.

20. Giltay EJ, Gooren LJ, Toorians AW, Katan MB, Zock PL. Docosahexaenoic acid concentrations are higher in women than in men because of estrogenic effects. Am J Clin Nutr 2004; 80: 1167-74.

21. Hurtado de Catalfo GE, de Gomez Dumm IN. Influence of testosterone on polyunsaturated fatty acid biosynthesis in Sertoli cells in culture. Cell Biochem Funct 2005; 23: 175-80.

22. Hulbert AJ, Pamplona R, Buffenstein R, Buttemer WA. Life and death: metabolic rate, membrane composition, and life span of animals. Physiol Rev 2007; 87: 1175-213.

23. Ordovas JM, Mooser V. Nutrigenomics and nutrigenetics. Curr Opin Lipidol 2004; 15: 101-8.

24. Ratnayake WM, Galli C. Fat and fatty acid terminology, methods of analysis and fat digestion and metabolism: a background review paper. Ann Nutr Metab 2009; 55: 8-43.

25. Bourre JM, Dumont O, Durand G. Dose-effect of dietary oleic acid: oleic acid is conditionally essential for some organs. Reprod Nutr Dev 2004; 44: 371-80.

26. Hirasawa A, Hara T, Katsuma S, Adachi T, Tsujimoto G. Free fatty acid receptors and drug discovery. Biol Pharm Bull 2008; 31: 1847-51.

27. Nakamura MT, Cho HP, Xu J, Tang Z, Clarke SD. Metabolism and functions of highly unsaturated fatty acids: an update. Lipids 2001; 36: 961-4.

28. Calder PC. Polyunsaturated fatty acids and inflammatory processes: new twists in an old tale. Biochimie 2009; 91: 791-5.

29. Brenner RR. The oxidative desaturation of unsaturated fatty acids in animals. Mol Cell Biochem 1974; 3: 41-52.

30. Nakamura MT, Nara TY. Structure, function, and dietary regulation of delta6, delta5, and delta9 desaturases. Annu Rev Nutr 2004; 24: 345-76.

31. Brenner RR, Peluffo RO. Effect of saturated and unsaturated fatty acids on the desaturation in vitro of palmitic, stearic, oleic, linoleic, and linolenic acids. J Biol Chem 1966; 241: 5213-9.
32. Holman RT. The slow discovery of the importance of omega 3 essential fatty acids in human health. J Nutr 1998; 128 (2 Suppl): 427S-33S.

33. Simopoulos AP. Evolutionary aspects of diet, the omega-6/omega-3 ratio and genetic variation: nutritional implications for chronic diseases. Biomed Pharmacother 2006; 60: 502-7.

34. Yehuda S, Carasso RL. Modulation of learning, pain thresholds, and thermoregulation in the rat by preparations of free purified alpha-linolenic and linoleic acids: determination of the optimal omega 3-to-omega 6 ratio. Proc Natl Acad Sci U S A 1993; 90: 10345-9.

35. Rapoport SI, Rao JS, Igarashi M. Brain metabolism of nutritionally essential polyunsaturated fatty acids depends on both the diet and the liver. Prostaglandins Leukot Essent Fatty Acids 2007; 77: 251-61.

36. Morse NL. A meta-analysis of blood fatty acids in people with learning disorders with particular interest in arachidonic acid. Prostaglandins Leukot Essent Fatty Acids 2009; 81: 373-89.

37. Nassar BA, Huang YS, Manku MS, Das UN, Morse N, Horrobin DF. The influence of dietary manipulation with $\mathrm{n}-3$ and $\mathrm{n}-6$ fatty acids on liver and plasma phospholipid fatty acids in rats. Lipids 1986; 21: 652-6.

38. Wang L, Folsom AR, Zheng ZJ, Pankow JS, Eckfeldt JH. Plasma fatty acid composition and incidence of diabetes in middle-aged adults: the Atherosclerosis Risk in Communities (ARIC) Study. Am J Clin Nutr 2003; 78 : 91-8.

39. Vessby B, Aro A, Skarfors E, Berglund L, Salminen I, Lithell $\mathrm{H}$. The risk to develop NIDDM is related to the fatty acid composition of the serum cholesterol esters. Diabetes 1994; 43: 1353-7.

40. Ohrvall M, Berglund L, Salminen I, Lithell H, Aro A, Vessby B. The serum cholesterol ester fatty acid composition but not the serum concentration of alpha tocopherol predicts the development of myocardial infarction in 50-year-old men: 19 years follow-up. Atherosclerosis 1996; 127: 65-71.

41. Miettinen TA, Naukkarinen V, Huttunen JK, Mattila S, Kumlin T. Fatty-acid composition of serum lipids predicts myocardial infarction. $\mathrm{Br}$ Med J (Clin Res Ed) 1982; 285: 993-6.

42. Wiberg B, Sundstrom J, Arnlov J, et al. Metabolic risk factors for stroke and transient ischemic attacks in middle-aged men: a community-based study with long-term follow-up. Stroke 2006; 37: 2898-903.

43. Sundstrom J, Lind L, Vessby B, Andren B, Aro A, Lithell H. Dyslipidemia and an unfavorable fatty acid profile predict left ventricular hypertrophy 20 years later. Circulation 2001; 103: 836-41.

44. Vessby B, Gustafsson IB, Tengblad S, Boberg M, Andersson $A$. Desaturation and elongation of fatty acids and insulin action. Ann N Y Acad Sci 2002; 967: 183-95.

45. Warensjo E, Riserus U, Vessby B. Fatty acid composition of serum lipids predicts the development of the metabolic syndrome in men. Diabetologia 2005; 48: 1999-2005.

46. Petersson H, Basu S, Cederholm T, Riserus U. Serum fatty acid composition and indices of stearoyl-CoA desaturase activity are associated with systemic inflammation: longitudinal analyses in middle-aged men. $\mathrm{Br}$ J Nutr 2008; 99: 1186-9

47. Warensjo E, Sundstrom J, Vessby B, Cederholm T, Riserus $U$. Markers of dietary fat quality and fatty acid desaturation as predictors of total and cardiovascular mortality: a population-based prospective study. Am J Clin Nutr 2008; 88: 203-9. 
48. Das UN. A defect in the activity of Delta6 and Delta5 desaturases may be a factor in the initiation and progression of atherosclerosis. Prostaglandins Leukot Essent Fatty Acids 2007; 76: 251-68.

49. Das UN. A defect in the activity of delta6 and delta5 desaturases may be a factor predisposing to the de velopment of insulin resistance syndrome. Prostaglandins Leukotrienes Essen Fatty Acids 2005; 72: 343-50.

50. Kahn SG, Wind S, Slocum A, Pfeffer D, Yacowitz $H$ A study of the hypocholesterolemic activity of the eth$\mathrm{yl}$ esters of the polyunsaturated fatty acids of cod liver oil in the chicken. II. Effect on serum and tissue cholesterol and aortic and coronary atherosclerosis. J Nutr 1963; 80: 414-24.

51. Kahn SG, Vandeputte J, Wind S, Yacowitz AD. A Study of the hypocholesterolemic activity of the ethyl esters of the polyunsaturated fatty acids of cod liver oil in the chicken. I. Effect on total cholesterol. J Nutr 1963; 80: 403-13.

52. Kahn SG. A study of the hypocholesterolemic activity of the ethyl esters of the polyunsaturated fatty acids of cod liver oil in the rat. J Nutr 1964; 83: 262-6.

53. Kingsbury KJ, Aylott C, Morgan DM, Emmerson R. Effects of ethyl arachidonate, cod-liver oil, and corn oil on the plasma-cholesterol level. A comparison in normal volunters. Lancet 1961; 1: 739-41.

54. De Groot AP, Reed SA. Influence of dietary cod-liver oil and some fractions of cod-liver oil on serum cholesterol-level of rats. Nature 1959; 183: 1191.

55. Hauge JG, Nicolaysen R. The serum cholesterol depressive effect of linoleic, linolenic acids and of cod liver oil in experimental hypercholesterolaemic rats. Acta Physiol Scand 1959; 45: 26-30.

56. Dasgupta S, Bhattacharyya DK. Dietary effect of gamma-linolenic acid on the lipid profile of rat fed erucic acid rich oil. J Oleo Sci 2007; 56: 569-77.

57. Das UN. Hemostatic vitamins and prostaglandins. Med J Australia 1982; 2: 316.

58. Das UN. Minerals, trace elements and vitamins interact with essential fatty acids and prostaglandins to prevent hypertension, thrombosis, hypercholesterolemia and atherosclerosis and their attendant complications. IRCS J Med Sci 1985; 13: 684-6.

59. Huang YS, Das UN Horrobin DF. Effect of dexamethasone on the distribution of essential fatty acids in plasma and liver phospholipids. IRCS Med Sci 1986 14: 180-1.

60. Das UN. Metabolic syndrome pathophysiology: the role of essential fatty acids and their metabolites. Wiley-Blackwell Publishers, Ames, IA, USA, 2010.

61. Das UN. Molecular basis of health and disease. Spring er, New York 2011.

62. Das UN. Folic acid and polyunsaturated fatty acids improve cognitive function and prevent depression, dementia, and Alzheimer's disease: but how and why? Prostaglandins Leukot Essent Fatty Acids 2008; 78: 11-9.

63. Das UN. Delta6 desaturase as the target of the beneficial actions of magnesium. Med Sci Monit 2010; 16: LE11-2.

64. Das UN. Essential fatty acids - a review. Curr Pharmaceut Biotechnol 2006; 7: 467-82

65. Das UN. Essential fatty acids: biochemistry, physiology, and pathology. Biotechnol J 2006; 1: 420-39.

66. Brenner RR. Nutritional and hormonal factors influencing desaturation of essential fatty acids. Prog Lipid Res 1981; 20: 41-7.

67. Le HD, Fallon EM, Kalish BT, et al. The effect of varying ratios of docosahexaenoic acid and arachidonic acid in the prevention and reversal of biochemical essential fatty acid deficiency in a murine model. Metabolism 2013; 62: 499-508.

68. Extier A, Perruchot MH, Baudry C, Guesnet P, Lavialle $M$, Alessandri JM. Differential effects of steroids on the synthesis of polyunsaturated fatty acids by human neuroblastoma cells. Neurochem Int 2009; 55: 295-301.

69. Burdge G. Alpha-linolenic acid metabolism in men and women: nutritional and biological implications. Curr Opin Clin Nutr Metab Care 2004; 7: 137-44.

70. Bakewell L, Burdge GC, Calder PC. Polyunsaturated fatty acid concentrations in young men and women consuming their habitual diets. Br J Nutr 2006; 96: 93-9.

71. Nakamura MT, Phinney SD, Tang AB, Oberbauer AM, German JB, Murray JD. Increased hepatic delta 6-desaturase activity with growth hormone expression in the MG101 transgenic mouse. Lipids 1996; 31: 139-43.

72. Zolfaghari R, Cifelli CJ, Banta MD, Ross AC. Fatty acid del$\mathrm{ta}(5)$-desaturase mRNA is regulated by dietary vitamin A and exogenous retinoic acid in liver of adult rats. Arch Biochem Biophys 2001; 391: 8-15.

73. Shimizu S, Akimoto K, Shinmen Y, Kawashima H, Sugano $M$, Yamada $H$. Sesamin is a potent and specific in hibitor of delta 5 desaturase in polyunsaturated fatty acid biosynthesis. Lipids 1991; 26: 512-6.

74. Leikin Al, Brenner RR. Fatty acid desaturase activities are modulated by phytosterol incorporation in micro somes. Biochim Biophys Acta 1989; 1005: 187-91.

75. Ves Losada A, Peluffo RO. Effect of cold environment on hepatic microsomal delta 6 and delta 9 desaturase activity of male rats. Lipids 1987; 22: 583-8.

76. Gonzalez S, Nervi AM, Peluffo RO, Brenner RR. Effect of environmental temperature changes on rat liver fatty acid desaturases. Lipids 1983; 18: 7-11.

77. Brodte E, Graeve M, Jacob U, Knust R, Pörtner HO. Temperature-dependent lipid levels and components in polar and temperate eelpout (Zoarcidae). Fish Physiol Biochem 2008; 34: 261-74.

78. Dinh TK, Bourre JM, Durand G. Effect of age and alpha-linolenic acid deficiency on delta 6 desaturase activity and liver lipids in rats. Lipids 1993; 28: 517-23.

79. Cho HP, Nakamura M, Clarke SD. Cloning, expression, and fatty acid regulation of the human delta-5 desaturase. J Biol Chem 1999; 274: 37335-9.

80. Chawla A, Repa JJ, Evans RM, Mangelsdorf DJ. Nuclear receptors and lipid physiology: opening the $\mathrm{X}$-files. Science 2001; 294: 1866-70.

81. Cheung GW, Kokorovic A, Lam TK. Upper intestinal lipids regulate energy and glucose homeostasis. Cell Mol Life Sci 2009; 66: 3023-7.

82. Lam CK, Chari M, Lam TK. CNS regulation of glucose homeostasis. Physiology (Bethesda) 2009; 24: 159-70.

83. Kua $\mathrm{CH}$. Uncoupling the relationship between fatty acids and longevity. IUBMB Life 2006; 58: 153-5.

84. Dougherty RM, Galli C, Ferro-Luzzi A, Iacono JM. Lipid and phospholipid fatty acid composition of plasma, red blood cells, and platelets and how they are affected by dietary lipids: a study of normal subjects from Italy, Finland, and the USA. Am J Clin Nutr 1987; 45: 443-55.

85. Andersson A, Nalsen C, Tengblad S, Vessby B. Fatty acid composition of skeletal muscle reflects dietary fat composition in humans. Am J Clin Nutr 2002; 76: 1222-9.

86. Raatz SK, Bibus D, Thomas W, Kris-Etherton P. Total fat intake modifies plasma fatty acid composition in humans. J Nutr 2001; 131: 231-4. 
87. Das UN. Essential fatty acids and their metabolites in the context of hypertension. Hypertension Res 2010; 33: 782-5.

88. Weisinger HS, Armitage JA, Sinclair AJ, Vingrys AJ, Burns PL, Weisinger RS. Perinatal omega-3 fatty acid deficiency affects blood pressure later in life. Nat Med 2001; 7: 258-9.

89. Begg DP, Sinclair AJ, Stahl LA, et al. Hypertension induced by omega-3 polyunsaturated fatty acid deficiency is alleviated by alpha-linolenic acid regardless of dietary source. Hypertens Res 2010; 33: 808-13.

90. Suryaprabha P, Das UN, Koratkar R, Sangeetha P, Ramesh $G$. Free radical generation, lipid peroxidation and essential fatty acids in uncontrolled hypertension. Prostaglandins Leukot Essen Fatty Acids 1990; 41: 27-33.

91. Das UN. Essential fatty acids and their metabolites could function as endogenous HMG-CoA reductase and ACE enzyme inhibitors, anti-arrhythmic, anti-hypertensive, anti-atherosclerotic, anti-inflammatory, cytoprotective, and cardioprotective molecules. Lipids Health Dis 2008; 7: 37.

92. Das UN. Interaction(s) of polyunsaturated fatty acids with dietary protein and its relationship to the pathogenesis of hypertension. Am J Hypertens 2010; 23: 111-2.

93. Buettner GR. The pecking order of free radicals and antioxidants: lipid peroxidation, alpha-tocopherol, and ascorbate. Arch Biochem Biophys 1993; 300: 535-43.

94. Turchetto E, Pignatti C. Protection of essential fatty acids by vitamin E. Acta Vitaminol Enzymol 1982; 4: 267-77.

95. Cabré E, Periago JL, Mingorance MD, et al. Factors related to the plasma fatty acid profile in healthy subjects, with special reference to antioxidant micronutrient status: a multivariate analysis. Am J Clin Nutr 1992; 55: 831-7.

96. Yilmaz O, Celik S, Naziroğlu M, Cay M, Dilsiz N. The effects of dietary selenium and vitamin $\mathrm{E}$ and their combination on the fatty acids of erythrocytes, bone marrow and spleen tissue lipids of lambs. Cell Biochem Funct 1997; 15: 1-7.

97. Meydani SN, Meydani M, Verdon CP, Shapiro AA, Blumberg JB, Hayes KC. Vitamin E supplementation suppresses prostaglandin E1(2) synthesis and enhances the immune response of aged mice. Mech Ageing Dev 1986; 34: 191-201.

98. Wu D, Mura C, Beharka AA, et al. Age-associated increase in PGE2 synthesis and COX activity in murine macrophages is reversed by vitamin E. Am J Physiol 1998; 275: C661-8.

99. Meydani SN, Wu D. Nutrition and age-associated inflammation: implications for disease prevention. JPEN J Parenter Enteral Nutr 2008; 32: 626-9.

100. Jiang Q, Ames BN. Gamma-tocopherol, but not alpha-tocopherol, decreases proinflammatory eicosanoids and inflammation damage in rats. FASEB J 2003; 17: 816-22.

101. Sravan Kumar G, Das UN, Vijay Kumar K, Madhavi, Das NP, Tan BKH. Effect of n- 6 and n-3 fatty acids on the proliferation and secretion of TNF and IL-2 by human lymphocytes in vitro. Nutrition Res 1992; 12: 815-23.

102. Sravan Kumar G, Das UN. Effect of prostaglandins and their precursors on the proliferation of human lymphocytes and their secretion of tumor necrosis factor and various interleukins. Prostaglandins Leukotrienes Essential Fatty Acids 1994; 50: 331-4.

103. Dietary supplementation with $n-3$ polyunsaturated fatty acids and vitamin $\mathrm{E}$ after myocardial infarction: results of the GISSI-Prevenzione trial. Gruppo Italiano per lo Studio della Sopravvivenza nell'Infarto miocardico. Lancet 1999; 354: 447-55.

104. Omenn GS. Chemoprevention of lung cancer: the rise and demise of beta-carotene. Annu Rev Public Health 1998; 19: 73-99.

105. Kampmann E, Johann S, van Neerven S, Beyer C, Mey J. Anti-inflammatory effect of retinoic acid on prostaglandin synthesis in cultured cortical astrocytes. J Neurochem 2008; 106: 320-32.

106. Hung LF, Lai JH, Lin LC, et al. Retinoid acid inhibits IL-1induced iNOS, COX-2 and chemokine production in human chondrocytes. Immunol Invest 2008; 37: 675-93.

107. Pita ML, Delgado MJ. Folate administration increases $\mathrm{n}-3$ polyunsaturated fatty acids in rat plasma and tissue lipids. Thromb Haemost 2000; 84: 420-3.

108. Candelario-Jalil E, de Oliveira AC, Graf S, et al. Resveratrol potently reduces prostaglandin E2 production and free radical formation in lipopolysaccharide-activated primary rat microglia. J Neuroinflammation 2007; 4: 25.

109. Bouhamidi R, Prevost V, Nouvelot A. High protection by grape seed proanthocyanidins (GSPC) of polyunsaturated fatty acids against UV-C induced peroxidation. C R Acad Sci III 1998; 321: 31-8.

110. Nonaka M, Yamashita K, lizuka Y, Namiki M, Sugano M. Effects of dietary sesaminol and sesamin on eicosanoid production and immunoglobulin level in rats given ethanol. Biosci Biotechnol Biochem 1997; 61: 836-9.

111. Catala A. The ability of melatonin to counteract lipid peroxidation in biological membranes. Curr Mol Med 2007; 7: 638-49.

112. Nishida S, Segawa T, Murai I, Nakagawa S. Long-term melatonin administration reduces hyperinsulinemia and improves the altered fatty-acid compositions in type 2 diabetic rats via the restoration of Delta-5 desaturase activity. J Pineal Res 2002; 32: 26-33.

113. Droge W. Free radicals in the physiological control of cell function. Physiol Rev 2002; 82: 47-95.

114. Spahis S, Vanasse M, Belanger SA, Ghadirian P, Grenier E, Levy E. Lipid profile, fatty acid composition and pro- and anti-oxidant status in pediatric patients with attention-deficit/hyperactivity disorder. Prostaglandins Leukot Essent Fatty Acids 2008; 79: 47-53.

115. Levy E, Rizwan Y, Thibault L, et al. Altered lipid profile, lipoprotein composition, and oxidant and antioxidant status in pediatric Crohn disease. Am J Clin Nutr 2000; 71: 807-15.

116. Cani PD, Delzenne NM. Gut microflora as a target for energy and metabolic homeostasis. Curr Opin Clin Nutr Metab Care 2007; 10: 729-34.

117. Turnbaugh PJ, Ley RE, Mahowald MA, Magrini V, Mardis ER, Gordon JI. An obesity-associated gut microbiome with increased capacity for energy harvest. Nature 2006; 444: 1027-31.

118. Wall R, Ross RP, Shanahan F, et al. Metabolic activity of the enteric microbiota influences the fatty acid composition of murine and porcine liver and adipose tissues. Am J Clin Nutr 2009; 89: 1393-401.

119. Hurtado de Catalfo GE, de Alaniz MJ, Marra CA. Influence of commercial dietary oils on lipid composition and testosterone production in interstitial cells isolated from rat testis. Lipids 2009; 44: 345-57.

120. Gromadzka-Ostrowska J. Effects of dietary fat on androgen secretion and metabolism. Reprod Biol 2006; 6 Suppl 2: 13-20. 
121. Das UN. Estrogen, statins, and polyunsaturated fatty acids: similarities in their actions and benefits - is there a common link? Nutrition 2002; 18: 178-88.

122. Meeker JD, Godfrey-Bailey L, Hauser R. Relationships between serum hormone levels and semen quality among men from an infertility clinic. J Androl 2007; 28: 397-406.

123. Blaak E. Gender differences in fat metabolism. Curr Opin Clin Nutr Metab Care 2001; 4: 499-502.

124. Williams CM. Lipid metabolism in women. Proc Nutr Soc 2004; 63: 153-60.

125. Childs CE, Romeu-Nadal M, Burdge GC, Calder PC. The polyunsaturated fatty acid composition of hepatic and plasma lipids differ by both sex and dietary fat intake in rats. J Nutr 2010; 140: 245-50.

126. Priego T, Sanchez J, Pico C, Palou A. Sex-differential expression of metabolism-related genes in response to a high-fat diet. Obesity (Silver Spring) 2008; 16: 819-26

127. Waxman DJ, Holloway MG. Sex differences in the expression of hepatic drug metabolizing enzymes. Mol Pharmacol 2009; 76: 215-28.

128. Lovegrove JA, Gitau R. Personalized nutrition for the prevention of cardiovascular disease: a future perspective. J Hum Nutr Diet 2008; 21: 306-16.

129. Simopoulos AP. The role of fatty acids in gene expression: health implications. Ann Nutr Metab 1996; 40: 303-11.

130. Sampath H, Ntambi JM. Polyunsaturated fatty acid regulation of genes of lipid metabolism. Annu Rev Nutr 2005; 25: 317-40.

131. Calpe-Berdiel L, Escola-Gil JC, Ribas V, Navarro-Sastre A, Garces-Garces J, Blanco-Vaca F. Changes in intestinal and liver global gene expression in response to a phytosterol-enriched diet. Atherosclerosis 2005 181: 75-85.

132. Moosmann B, Behl C. Secretory peptide hormones are biochemical antioxidants: structure-activity relationship. Mol Pharmacol 2002; 61: 260-8.

133. Brenner RR. Effect of unsaturated acids on membrane structure and enzyme kinetics. Prog Lipid Res 1984; 23: 69-96.

134. Armstrong VT, Brzustowicz MR, Wassall SR, Jenski LJ, Stillwell W. Rapid flip-flop in polyunsaturated (docosahexaenoate) phospholipid membranes. Arch Biochem Biophys 2003; 414: 74-82.

135. Yu BP. Membrane alteration as a basis of aging and the protective effects of calorie restriction. Mech Ageing Dev 2005; 126: 1003-10.

136. Urano S, Sato Y, Otonari T, et al. Aging and oxidative stress in neurodegeneration. Biofactors 1998; 7: 103-12.

137. Fukui K, Omoi NO, Hayasaka T, et al. Cognitive impairment of rats caused by oxidative stress and aging, and its prevention by vitamin E. Ann N Y Acad Sci 2002 959: 275-84.

138. Li Y, Nara TY, Nakamura MT. Peroxisome proliferator-activated receptor alpha is required for feedback regulation of highly unsaturated fatty acid synthesis. J Lipid Res 2005; 46: 2432-40.

139. Biagi PL, Bordoni A, Hrelia S, Celadon M, Horrobin DF. Gamma-linolenic acid dietary supplementation can reverse the aging influence on rat liver microsome delta 6-desaturase activity. Biochim Biophys Acta 1991; 1083: 187-92.

140. van Dooremalen C, Pel R, Ellers J. Maximized PUFA measurements improve insight in changes in fatty acid composition in response to temperature. Arch Insect Biochem Physiol 2009; 72: 88-104.

141. Harbige LS. Fatty acids, the immune response, and autoimmunity: a question of n- 6 essentiality and the balance between n-6 and n-3. Lipids 2003; 38: 323-41.
142. Ergas D, Eilat E, Mendlovic S, Sthoeger ZM. n-3 fatty acids and the immune system in autoimmunity. Isr Med Assoc J 2002; 4: 34-8.

143. Surh YJ. NF-kappa B and Nrf2 as potential chemopreventive targets of some anti-inflammatory and antioxidative phytonutrients with anti-inflammatory and antioxidative activities. Asia Pac J Clin Nutr 2008; 17 Suppl 1: 269-72.

144. Boland LM. Polyunsaturated fatty acid modulation of voltage-gated ion channels. Cell Biochem Biophys 2008; 52: 59-84.

145. Kiecolt-Glaser JK, Belury MA, Porter K, Beversdorf DQ Lemeshow S, Glaser R. Depressive symptoms, omega-6 : omega-3 fatty acids, and inflammation in older adults. Psychosom Med 2007; 69: 217-24.

146. Tassoni D, Kaur G, Weisinger RS, Sinclair AJ. The role of eicosanoids in the brain. Asia Pac J Clin Nutr 2008; 17 Suppl 1: 220-8.

147. Gerashchenko D, Okano Y, Urade Y, Inoue S, Hayaishi O. Strong rebound of wakefulness follows prostaglandin D2- or adenosine A2a receptor agonist-induced sleep. J Sleep Res 2000; 9: 81-7.

148. Onoe H, Ueno R, Fujita I, Nishino H, Oomura Y, Hayaishi O. Prostaglandin D2, a cerebral sleep-inducing substance in monkeys. Proc Natl Acad Sci U S A 1988; 85: 4082-6.

149. Krueger JM. The role of cytokines in sleep regulation. Curr Pharm Des 2008; 14: 3408-16.

150. Dinan T, Siggins L, Scully P, O'Brien S, Ross P, Stanton $C$. Investigating the inflammatory phenotype of major depression: focus on cytokines and polyunsaturated fatty acids. J Psychiatr Res 2009; 43: 471-6.

151. Robertson MD, Jackson KG, Fielding BA, Williams CM, Frayn KN. Acute effects of meal fatty acid composition on insulin sensitivity in healthy post-menopausal women. Br J Nutr 2002; 88: 635-40.

152. de Wilde J, Mohren R, van den Berg S, et al. Short-term high fat-feeding results in morphological and metabolic adaptations in the skeletal muscle of C57BL/6J mice. Physiol Genomics 2008; 32: 360-9.

153. Stępień M, Stępień A, Wlazeł RN, Paradowski M, Banach $M$, Rysz J. Obesity indices and inflammatory markers in obese non-diabetic normo- and hypertensive patients: a comparative pilot study. Lipids Health Dis 2014; 13: 29.

154. Rizzo M, Banach M, Montalto G, Mikhailidis DP. Lipid-lowering therapies and achievement of LDL-cholesterol targets. Arch Med Sci 2012; 8: 598-600.

155. Otocka-Kmiecik A, Mikhailidis DP, Nicholls SJ, Davidson M, Rysz J, Banach M. Dysfunctional HDL: a novel important diagnostic and therapeutic target in cardiovascular disease? Prog Lipid Res 2012; 51: 314-24.

156. Mariscalco G, Sarzi Braga S, Banach M, et al. Preoperative $n-3$ polyunsaturated fatty acids are associated with a decrease in the incidence of early atrial fibrillation following cardiac surgery. Angiology 2010; 61: 643-50.

157. Cicero AF, Reggi A, Parini A, Borghi C. Application of polyunsaturated fatty acids in internal medicine: beyond the established cardiovascular effects. Arch Med Sci 2012; 8: 784-93.

158. Das UN. Essential fatty acids as possible mediators of the actions of statins. Prostaglandins Leukot Essent Fatty Acids 2001; 65: 37-40.

159. Rizzo M, Nikolic D, Banach M, Montalto G. Statin treatment in the elderly: how much do we know? Arch Med Sci 2013; 9: 585-8. 